\title{
Evaluation of prognostic models developed using standardised image features from different PET automated segmentation methods
}

Craig Parkinson ${ }^{1}$, Kieran Foley ${ }^{2^{*}}$ D, Philip Whybra ${ }^{1}$, Robert Hills ${ }^{3}$, Ashley Roberts ${ }^{4}$, Chris Marshall ${ }^{5}$, John Staffurth ${ }^{2,6}$ and Emiliano Spezi ${ }^{1,6}$

\begin{abstract}
Background: Prognosis in oesophageal cancer (OC) is poor. The 5-year overall survival (OS) rate is approximately $15 \%$. Personalised medicine is hoped to increase the 5- and 10-year OS rates. Quantitative analysis of PET is gaining substantial interest in prognostic research but requires the accurate definition of the metabolic tumour volume. This study compares prognostic models developed in the same patient cohort using individual PET segmentation algorithms and assesses the impact on patient risk stratification.

Consecutive patients $(n=427)$ with biopsy-proven OC were included in final analysis. All patients were staged with PET/ CT between September 2010 and July 2016. Nine automatic PET segmentation methods were studied. All tumour contours were subjectively analysed for accuracy, and segmentation methods with < $90 \%$ accuracy were excluded. Standardised image features were calculated, and a series of prognostic models were developed using identical clinical data. The proportion of patients changing risk classification group were calculated.

Results: Out of nine PET segmentation methods studied, clustering means (KM2), general clustering means (GCM3), adaptive thresholding (AT) and watershed thresholding (WT) methods were included for analysis. Known clinical prognostic factors (age, treatment and staging) were significant in all of the developed prognostic models. AT and KM2 segmentation methods developed identical prognostic models. Patient risk stratification was dependent on the segmentation method used to develop the prognostic model with up to 73 patients (17.1\%) changing risk stratification group.

Conclusion: Prognostic models incorporating quantitative image features are dependent on the method used to delineate the primary tumour. This has a subsequent effect on risk stratification, with patients changing groups depending on the image segmentation method used.
\end{abstract}

Keywords: Prognostic model, Esophageal cancer, PET/CT, Automated segmentation

\footnotetext{
*Correspondence: foleykg@cardiff.ac.uk

${ }^{2}$ Division of Cancer and Genetics, School of Medicine, UHW Main Building,

Heath Park, Cardiff CF14 4XN, UK

Full list of author information is available at the end of the article
} 


\section{Highlights}

- Texture features are dependent on the segmentation method

- Prognostic scores differ between models derived using different segmentation methods

- Patient risk stratification using identical clinical data is dependent on the segmentation method

\section{Background}

Prognosis in oesophageal cancer (OC) is poor. The 1- and 5 -year overall survival (OS) rate is 44 and $15 \%$, respectively [1]. The aim of precision medicine and prognostic models is to ensure each patient is managed with the most appropriate treatment, which may improve patient OS [2-4]. The avoidance of futile aggressive therapies prevents unnecessary treatment and improves quality of life. In addition, better patient stratification may also allow more efficient trial designs.

Prognostic models are formulated from patient specific information such as age, pathological subtype, molecular characterisation and tumour staging. However, the advanced quantitative analysis of medical images, especially CT, MR and PET, is gaining substantial interest in prognostic research as more accurate prognostic models may be developed. Radiomic features characterise tumour phentotypes through extraction of high-dimensional data [5] and can be associated with metastatic growth, recurrence and survival in several solid cancers [6]. These methods may also have added prognostic value in cancer staging pathways [7].

The accurate delineation of the relevant metabolic tumour volume (MTV) on PET/CT is challenging due to low spatial resolution and the high noise characteristics of PET imaging [8]. Many different PET segmentation techniques have been proposed as a solution to the delineation of the MTV [9]. Numerous PET-based radiomic features have been described, but the results of radiomic analysis are highly dependent on the method used to derive the MTV [10]. Few studies have compared results of radiomic analysis derived from each segmentation method (cf. [11] and references therein) or have investigated their effect on patient risk stratification derived from prognostic models [12-14].

This study aimed to develop a series of prognostic models in the same patient cohort using identical clinical data and standardised radiomic features derived from different segmentation methods. The impact of using different segmentation methods on patient risk stratification was assessed.

\section{Methods}

\section{Patient cohort}

This is a retrospective cohort study of consecutive patients with biopsy-proven OC, including gastro-oesophageal junctional (GOJ) tumours, radiologically staged between 16 September 2010 and 31 July 2016. Patients were identified from a database of $\mathrm{OC}$ patients used in a previous study [15]. Institutional Review Board approval was granted and requirement for informed consent was waived (Wales REC 1, UK reference 14/WA/1208).

Overall, 486 patients with FDG-avid primary oesophageal and GOJ tumours were considered for inclusion. Fourteen patients were excluded due to missing clinical data. All patients were deemed to have potentially curable disease following contrast-enhanced CT staging investigation. All $\mathrm{PET} / \mathrm{CT}$ examinations were performed separately, following the initial CT, and reported in the same centre by Consultant Radiologists with an interest in Nuclear Medicine. Radiological staging was performed according to the International Union Against Cancer (UICC) TNM 7th edition [16]. Following exclusions, 472 patients were studied.

\section{PET/CT protocol}

Patients were fasted for at least $6 \mathrm{~h}$ prior to tracer administration. Serum glucose levels were routinely checked and confirmed as less than $7.0 \mathrm{mmol} / \mathrm{L}$ prior to imaging. Patients received a dose of $4 \mathrm{MBq}$ of ${ }^{18} \mathrm{~F}-\mathrm{FDG} / \mathrm{kg}$. Uptake time was $90 \mathrm{~min}$, standard practice at our institution. A GE 690 scanner (GE Healthcare, Buckinghamshire, UK) was used. CT images were acquired in a helical acquisition with a pitch of 0.98 and tube rotation speed of $0.5 \mathrm{~s}$. Tube output was $120 \mathrm{kVp}$ with output modulation between 20 and $200 \mathrm{~mA}$. Matrix size for the CT acquisition was $512 \times 512$ pixels with a $50 \mathrm{~cm}$ field of view. No oral or intravenous contrast was administered. PET images were acquired at $3 \mathrm{~min}$ per field of view. The length of the axial field of view was $15.7 \mathrm{~cm}$ (skull base to mid-thigh). Images were reconstructed with the ordered subset expectation maximisation algorithm, with 24 subsets and 2 iterations. Matrix size was $256 \times 256$ pixels, using the VUE Point ${ }^{\text {tax }}$ time of flight algorithm. All PET-based data was obtained using the same PET/CT scanner and reconstruction method with voxel dimensions of $2.73 \times 2.73 \times 3.27 \mathrm{~mm}$.

\section{Treatment protocols}

Patients began treatment 2-4 weeks after staging FDG PET/CT imaging. Patients either had endoscopic mucosal resection (EMR), surgery alone, neo-adjuvant chemotherapy (NACT) or neo-adjuvant chemoradiotherapy (NACRT) prior to surgery, definitive chemo-radiotherapy (dCRT) or palliative therapy. The optimum treatment strategy was decided by consensus at the MDT. In general, fit patients with tumours pre-operatively staged as T3/T4a, N0/N1 were pre-operatively treated with NACT or NACRT. Less fit patients, or those with T1/2 N0 disease, had surgery alone. Patients deemed unsuitable for surgery due to comorbidity and/or performance status, extensive locoregional disease, or personal choice received dCRT. 


\section{Data preparation and PET segmentation}

Manual delineation of the metabolic tumour volume (MTV) is limited by intra- and inter-observer variability and is time consuming [17-19]. Semi-automated and automated segmentation methods are favourable alternatives by reducing variability in delineation and decreasing the contouring time [20]. Fixed percentage thresholding has been shown to be dependent upon the $\mathrm{SUV}_{\max }$ of a tumour as well as the MTV [21]. Furthermore, it has been shown that texture analysis of PET imaging is dependent upon the segmentation method used to define the MTV [12, 22, 23]. However, more complex segmentation algorithms such as adaptive iterative thresholding (AT) have been shown to be independent of SUV ${ }_{\max }$ as well as being correlated to the MTV. Segmentation methods adopting clustering techniques such as Fuzzy C-means (FCM), Gaussian fuzzy Cmeans (GCM) and K-means (KM) using 2, 3 and 4 clusters (FCM2, GCM3-4, KM2 - KM4), as well as region growing (RG) and watershed transform (WT) methods, are promising segmentation methods in the delineation of the MTV. These segmentation methods are reviewed in detail in the report by Hatt et al. [9], are described in detail previously [24] and are summarised in Table 1 . In each case, the MTV was defined using AT, FCM2, GCM3, GCM4, KM2, KM3, KM4, RG and WT PET segmentation methods.

A clinical radiologist subjectively assessed each tumour contour produced by all nine PET segmentation methods for accurate tumour representation. All tumour contours were visualised using the same software and image settings to ensure consistent methodology. Segmentation methods were considered inadequate for further analysis if less than $90 \%$ of contours were non-representative. This pre-defined value was decided upon prior to image visualisation. Contours were assessed individually and classified as not representative if contours were greatly different from the primary tumour, or included bone, lung or medistinial tissue. In addition, segmentation methods that had failed

Table 1 Name and description of PET-AS methods used in this study, with references of published work using similar segmentation approaches

\begin{tabular}{lll}
\hline Algorithm & Description & Key references \\
\hline AT & $\begin{array}{l}\text { 3D adaptive iterative thresholding, } \\
\text { using background subtraction }\end{array}$ & $\begin{array}{l}\text { Jentzen et al. [43], } \\
\text { Drever et al. [44] }\end{array}$ \\
RG & $\begin{array}{l}\text { 3D region-growing with automatic } \\
\text { seed finder and stopping criterion }\end{array}$ & Day et al. [45] \\
KM & $\begin{array}{l}\text { 3D K-mean iterative clustering with } \\
\text { custom stopping criterion }\end{array}$ & $\begin{array}{l}\text { Zaidi and } \\
\text { El Naqa [8] }\end{array}$ \\
FCM & $\begin{array}{l}\text { 3D fuzzy C-mean iterative clustering } \\
\text { with custom stopping criterion }\end{array}$ & $\begin{array}{l}\text { Belhassen and } \\
\text { Zaidi [46] }\end{array}$ \\
GCM & $\begin{array}{l}\text { 3D Gaussian mixture models based } \\
\text { clustering with custom stopping criterion }\end{array}$ & Hatt et al. [37] \\
WT & $\begin{array}{l}\text { Watershed transform-based algorithm, } \\
\text { using sobel filter }\end{array}$ & $\begin{array}{l}\text { Geets et al. [47], } \\
\text { Tylski et al. [48] }\end{array}$ \\
\hline
\end{tabular}

and conformed to the boundary of the bounding box were defined as not representative of the primary tumour.

\section{Clinical data and image analysis}

Only primary tumours were analysed to ensure consistent methodology across all patients. Before quantitative image analysis and texture feature extraction, PET images were re-sampled into $0.5 \mathrm{SUV}$ bins. A fixed bin width maintains a constant intensity resolution when compared to approaches based on a fixed number of bins [25]. In the development of the prognostic models, age at diagnosis (number of years), radiological stage (stage IA-IV) and treatment (curative vs palliative) were included because these are strong predictors of survival [26]. Curative and palliative treatments were coded as 1 and 2 respectively. Radiological staging was modelled categorically.

Radiomic analysis was performed using features implemented as part of the Image Biomarker Standardisation Initiative (IBSI), a multicentre, international collaboration aimed at improving the reproducibility and validation of quantitative medical image analysis studies [5]. The radiomic features selected for inclusion in this study were chosen as they have shown prognostic and predictive significance in other radiomic studies investigating OC $[12,27,28]$. These have been summarised in Table 2 . Moreover, many radiomic feature implementations have been described $[6,7,27,29]$ and are divided into three groups for which a summary is provided. In this study, the MTV was analysed as a 3D volume with no thresholding applied to the MTV mask.

Table 2 Summary of quantitative imaging features

\begin{tabular}{|c|c|c|}
\hline $\begin{array}{l}\text { Type/order } \\
\text { of statistics }\end{array}$ & Feature & Brief definition \\
\hline Morphological & Volume & $\begin{array}{l}\text { Sum of voxels delineated multiplied by } \\
\text { the volume of one voxel }\end{array}$ \\
\hline \multirow[t]{2}{*}{ Pre-discretisation } & $\mathrm{SUV}_{\max }$ & Maximum uptake of FDG in the MTV \\
\hline & Energy & Sum squared SUV values in the MTV \\
\hline \multirow[t]{3}{*}{ First order } & Skewness & $\begin{array}{l}\text { Measures symmetry of intensity } \\
\text { histogram }\end{array}$ \\
\hline & Kurtosis & Measures flatness of intensity histogram \\
\hline & Entropy & Measures randomness \\
\hline Second order & Dissimilarity & $\begin{array}{l}\text { Variation of grey level pairs (GLCM). } \\
\text { Features were calculated for each } \\
\text { unique direction and averaged with } \\
\text { a distance setting of } 1\end{array}$ \\
\hline \multirow[t]{3}{*}{ Higher order } & $\begin{array}{l}\text { Grey-level } \\
\text { non-uniformity }\end{array}$ & $\begin{array}{l}\text { Distribution of zone counts for each } \\
\text { intensity value (GLSZM) }\end{array}$ \\
\hline & $\begin{array}{l}\text { Zone } \\
\text { percentage }\end{array}$ & $\begin{array}{l}\text { Fraction of recorded zones compared } \\
\text { to maximum possible }\end{array}$ \\
\hline & Coarseness & $\begin{array}{l}\text { Measures spatial rate of change in } \\
\text { intensity using a distance of } 1\end{array}$ \\
\hline
\end{tabular}




\section{First-order metrics}

First-order statistical metrics summarise the voxel intensity distribution within the segmented MTV, without concern for spatial relationships [30]. First-order metrics are typically histogram based and reduce the MTV to singular values describing the mean, minimum, maximum, median, and uniformity of the intensities within the MTV. Included in first-order stastical analysis is Skewness (asymmetry measure), Kurtosis (pointiness measure) and Entropy (randomness measure). Kurtosis and skewness have been shown to be independent predictors of survival [15] and of prognostic significance in the literature [31].

\section{Higher-order metrics}

Higher-order statistical metrics retain spatial information and are used to quantify inter-voxel intensity relationships. Dissimilarity is the quantification of variation in voxel pairs and is calculated using a Grey Level Co-occurrence Matrix (GLCM) generated for each unique direction and averaged. A low dissimilarity is resultant of neighbouring voxels having similar values [32]. Zone percentage is calculated from a Grey Level Size Zone matrix (GLSZM) by assessing the fraction of recorded zones compared to the maximum number of possible zones. Heterogeneous MTVs have high zone percentage scores. Grey Level NonUniformity (GLNU) is an evaluation of the distribution of zone counts for each intensity value. The feature value is low when the number of zones associated with each intensity value are similar. Coarseness is a neighbourhood greytone difference matrix (NGTDM) feature that gives an indication of the level of spatial rate of change in intensity [33]. GLCM, GLSZM and NGTDM can be computed in $2 \mathrm{D}$ or $3 \mathrm{D}$. The matrices in this study were computed in $3 \mathrm{D}$ as this may highlight the multi-scale, directional properties of tumour tissue [34].

\section{Outcome data}

The primary outcome of the study was OS, defined as number of months survived from date of diagnosis. Patients were followed up 3-monthly for the first year, 6monthly until 5 years then annually thereafter, or until death. All included patients were followed up for at least 12 months. Date of death was obtained from the Cancer Network Information System Cymru database (CaNISC, Velindre NHS Trust, Wales).

\section{Statistical analysis}

Categorical variables were described as frequency (percent) and continuous variables as median (range) and differences assessed with appropriate non-parametric tests. Cumulative survival was calculated by the Kaplan-Meier life-table method. Cox regression models with backward conditional method were constructed using identical clinical data and imaging data derived from each of the segmentation methods. An individual prognostic score was calculated from each segmentation method by summation of the products of variables and their corresponding parameter estimate. Using this score, patients were separated into low, intermediate and high-risk groups (higher prognostic score deemed higher risk) and a log-rank test evaluated significant differences in OS. The number of patients that changed risk stratification group depending on the segmentation method used was calculated, and the OS for the different risk groups between models was analysed. A $p$ value of $<0.05$ was considered statistically significant. Statistical analysis was performed using SAS version 9.4 (SAS, NC, USA) and SPSS version 23.0 (IBM, Chicago, USA). Imaging data, software and delineated MTVs are not available publicly.

\section{Results}

Four hundred and seventy-two patients, each with nine MTV contours delineated by AT, FCM2, GCM3, GCM4, KM2, KM3, KM4, RG and WT PET segmentation methods were assessed by a Clinical Radiologist with 5 years of research experience for accurate tumour representation. Forty-five patients and five segmentation methods were excluded due to poor MTV delineation. FCM2 failed to delineate an acceptable tumour representation in $145(30.8 \%)$ of cases. KM3 and KM4 failed in $88(18.6 \%)$ and $215(45.6 \%)$ of cases, respectively. RG failed in 389 (82.5\%), and GCM4 in 33 (7\%) of cases. Therefore, 427 cases with MTVs delineated with KM2, GCM3, AT and WT PET segmentation methods deemed to have accurate tumour representation and included for further analysis.

The 427 cases included for analysis were used to develop the prognostic models for KM2, WT, GCM3 and AT methods. Baseline characteristics of patients are detailed in Table 3. The median OS of the cohort was 17.0 months (95\% confidence interval (95\% CI) 14.8-19.2). Median follow-up was 35.0 months (95\% CI 28.7-41.3). Overall 1and 2-year survival in the development cohort was $65.3 \%$ and $30.1 \%$, respectively.

\section{Development of prognostic models}

The final steps of each prognostic model are presented in Table 4. Three known clinical prognostic factors (age, radiological stage and treatment) remained in each derived model, but there was a difference in the inclusion of texture metrics by segmentation technique. AT and KM2 produced the same model output. Interestingly, IBSI metrics were not included in the final models for these segmentation methods. However, skewness and kurtosis were independently significant for survival using GCM3 method. Skewness and GLNU were significant using WT method. Their inclusion in the models illustrates their 
Table 3 Baseline characteristics of patient cohort

\begin{tabular}{|c|c|}
\hline Median age & 67.0 years (range $24-84$ ) \\
\hline Gender & Male 315 (73.8):female 112 (26.2) \\
\hline \multicolumn{2}{|l|}{ Histology } \\
\hline Adenocarcinoma & $313(73.3)$ \\
\hline Squamous cell carcinoma & $100(23.4)$ \\
\hline Undifferentiated & $5(1.2)$ \\
\hline High-grade dysplasia & $4(0.9)$ \\
\hline Neuro-endocrine & $3(0.7)$ \\
\hline Small cell carcinoma & $1(0.2)$ \\
\hline Sarcoma & $1(0.2)$ \\
\hline \multicolumn{2}{|l|}{ Tumour location } \\
\hline Oesophagus & $268(62.8)$ \\
\hline Upper third & $14(5.2)$ \\
\hline Middle third & $71(26.5)$ \\
\hline Lower third & $183(68.3)$ \\
\hline Gastro-oesophageal junction & $159(37.2)$ \\
\hline Siewert I & $67(42.1)$ \\
\hline Siewert II & $42(26.4)$ \\
\hline Siewert III & $50(31.4)$ \\
\hline \multicolumn{2}{|l|}{ Stage group } \\
\hline IA & $10(2.3)$ \\
\hline IB & $17(4.0)$ \\
\hline$\| \mathrm{A}$ & $70(16.4)$ \\
\hline$\| \mathrm{B}$ & $13(3.0)$ \\
\hline$\| \mathrm{A}$ & $97(22.7)$ \\
\hline$\| I \mathrm{~B}$ & $52(12.2)$ \\
\hline IIIC & $76(17.8)$ \\
\hline IV & $92(21.5)$ \\
\hline \multicolumn{2}{|l|}{ Treatment } \\
\hline Curative & $224(52.5)$ \\
\hline NACT & $86(38.4)$ \\
\hline dCRT & $86(38.4)$ \\
\hline Surgery alone & $31(13.8)$ \\
\hline NACRT & $20(8.9)$ \\
\hline EMR & $1(0.5)$ \\
\hline Palliative & $203(47.5)$ \\
\hline \multicolumn{2}{|l|}{ Mortality } \\
\hline Alive & $132(30.9)$ \\
\hline Dead & $295(69.1)$ \\
\hline
\end{tabular}

additional prognostic value compared with current prognostic factors.

\section{Prognostic score calculation}

The equations for each model derived from different segmentation methods were used to calculate the prognostic
Table 4 Final output of prognostic models derived using AT, GCM3, KM2 and WT PET segmentation methods

\begin{tabular}{lllll}
\hline & Parameter estimate & $p$ value & Hazard ratio & $95 \% \mathrm{Cl}$ \\
\hline AT & & & & \\
Age & 0.020 & 0.001 & 1.020 & $1.008-1.033$ \\
Treatment & -1.075 & $<0.001$ & 0.341 & $0.254-0.459$ \\
Stage & 0.144 & $<0.001$ & 1.155 & $1.072-1.245$ \\
GCM3 & & & & \\
Age & 0.019 & 0.003 & 1.019 & $1.006-1.032$ \\
Treatment & -1.024 & $<0.001$ & 0.359 & $0.266-0.485$ \\
Stage & 0.142 & $<0.001$ & 1.153 & $1.068-1.245$ \\
Kurtosis & 0.632 & 0.002 & 1.882 & $1.260-2.809$ \\
Skewness & -0.789 & 0.044 & 0.454 & $0.211-0.980$ \\
KM2 & & & & \\
Age & 0.020 & 0.001 & 1.020 & $1.008-1.033$ \\
Treatment & -1.075 & $<0.001$ & 0.341 & $0.254-0.459$ \\
Stage & 0.144 & $<0.001$ & 1.155 & $1.072-1.245$ \\
WT & & & & \\
Age & 0.018 & 0.004 & 1.018 & $1.006-1.031$ \\
Treatment & -1.063 & $<0.001$ & 0.345 & $0.257-0.464$ \\
Stage & 0.140 & $<0.001$ & 1.150 & $1.065-1.242$ \\
GLNU & 0.017 & 0.006 & 1.017 & $1.005-1.029$ \\
Skewness & 0.674 & 0.030 & 1.962 & $1.067-3.607$ \\
\hline & & & &
\end{tabular}

scores, and are listed in Table 5. These calculations were derived using published methods [35].

Figure 1 shows the risk stratification for WT, KM2, AT and GCM3. Median OS for the low-risk, intermediate-risk and high-risk groups in the AT- and KM2-derived prognostic model was 36.0 months (29.9-42.1 months), 18.0 months (15.1-20.9 months) and 9.0 months (7.810.2 months), respectively. Median OS for the low-risk, intermediate-risk and high-risk groups in the GCM3derived prognostic model was 36.0 months (28.843.2 months), 18.0 months (15.4-20.6 months) and 9.0 months (7.7-19.2 months). Median OS for the WT derived prognostic model low-risk, intermediate-risk and high-risk groups was 36 months (27.8-44.2 months), 19 months (15.1-23 months) and OS for the high-risk group was 9 months (7.7-10.3 months) respectively.

Table 5 Prognostic model equations

\begin{tabular}{ll}
\hline $\begin{array}{l}\text { Segmentation } \\
\text { Method }\end{array}$ & Prognostic model equation \\
\hline AT & $($ Age $\times 0.020-($ Treatment $\times 1.075)+($ Stage $\times 0.144)$ \\
GCM3 & $($ Age $\times 0.019)-($ Treatment $\times 1.024)+($ Stage $\times 0.142)$ \\
& $-($ Skewness $\times 0.789)+($ Kurtosis $\times 0.632)$ \\
KM2 & $($ Age $\times 0.020)-($ Treatment $\times 1.075)+($ Stage $\times 0.144)$ \\
WT & $($ Age $\times 0.018)-($ Treatment $\times 1.063)+($ Stage $\times 0.140)$ \\
& $+($ Skewness $\times 0.674)+($ GLNU $\times 0.017)$ \\
\hline
\end{tabular}



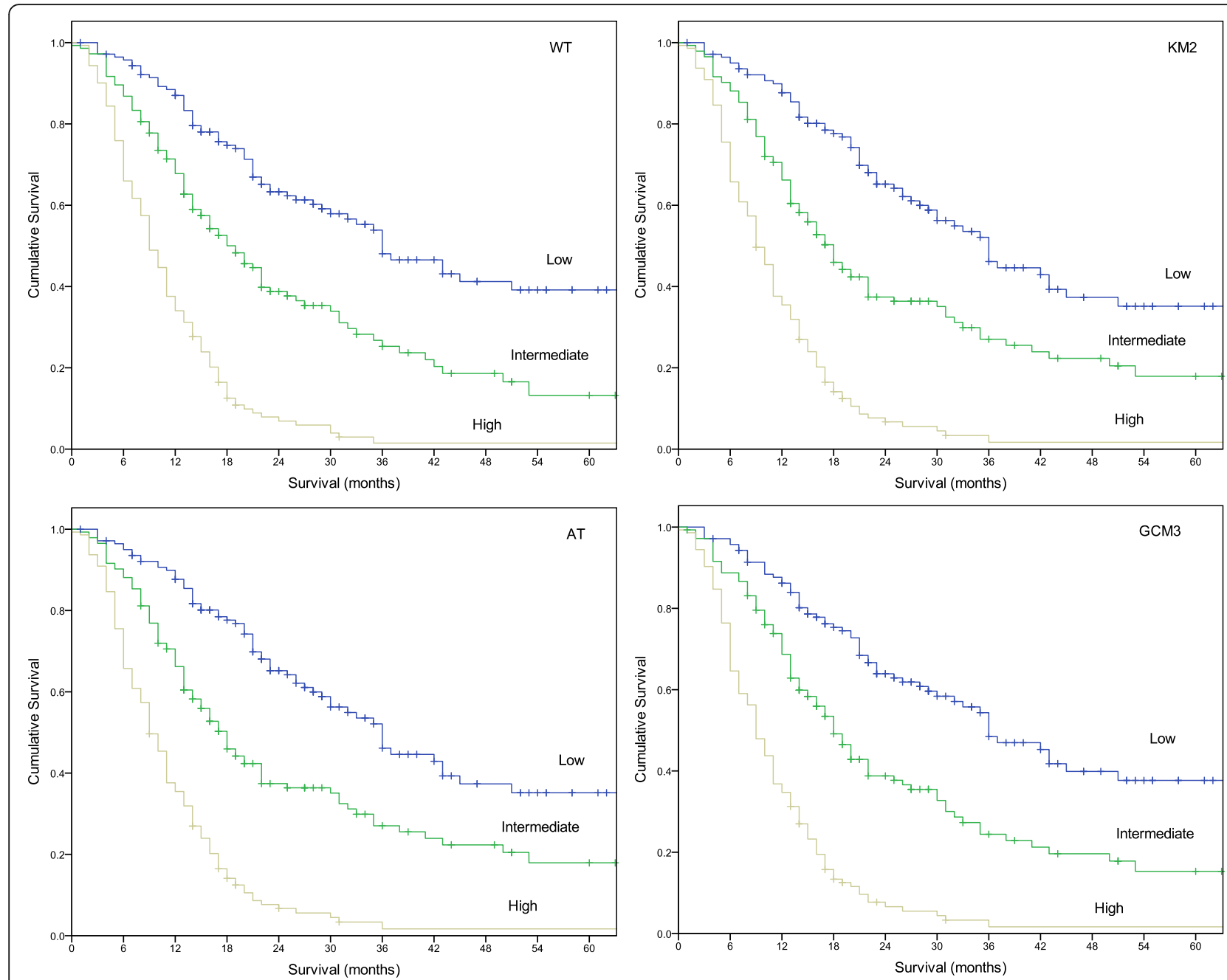

Fig. 1 Risk stratification and OS for WT (top left), KM2 (top right), AT (bottom left) GCM3 (bottom right)

Table 6 shows the number of patients stratified as low, intermediate and high-risk for each single prognostic model along with the prognostic score range for each risk stratification group. Table 7 shows the number of patients whom change risk stratification.

The largest proportion of patients to change risk stratification group was between prognostic models based on GCM3 and on WT ( $n=73,17.1 \%)$. It can be noted that no patient changed risk stratification group between AT and KM2 because the prognostic models were identical.

Table 6 Number of patients in each risk stratification group for each single prognostic model and prognostic score range

\begin{tabular}{llll}
\hline $\begin{array}{l}\text { Number of patients } \\
\text { in risk group } \\
\text { (prognostic range) }\end{array}$ & Low-risk & Intermediate-risk & High-risk \\
\hline AT/KM2 & $141(-0.45-0.98)$ & $143(0.99-2.16)$ & $143(2.17-2.79)$ \\
GCM3 & $140(-1.13-0.36)$ & $143(0.37-1.54)$ & $144(1.55-2.73)$ \\
WT & $142(-0.17-1.30)$ & $144(1.31-2.48)$ & $141(2.49-3.62)$ \\
\hline
\end{tabular}

The number of concordant patients stratified as low, intermediate and high-risk across the developed models was $118(28 \%), 95(22 \%)$ and 116 (27\%), respectively. There was no overall survival difference between AT, GCM3, KM2 or WT low-risk groups $\left(\chi^{2} 0.052\right.$, df 3, $p=0.997)$, intermediate-risk groups $\left(\chi^{2} 0.016\right.$, df 3 , $p=0.999)$ or high-risk groups $\left(\chi^{2} 0.028\right.$, df $\left.3, p=0.999\right)$.

For interest, Additional file 1 describes the developed prognostic models for the excluded PET-AS methods. Additional file 2 describes variances in radiomic features

Table 7 Total number of patients and percentage that change risk-stratification group

\begin{tabular}{lllll}
\hline Number changing group (\%) & AT & GCM3 & KM2 & WT \\
\hline AT & & & \\
GCM3 & $66(15.4)$ & & \\
KM2 & $0(0.0)$ & $66(15.4)$ & \\
WT & $57(13.3)$ & $73(17.1)$ & $57(13.3)$ & \\
\hline
\end{tabular}


extracted using differing discretisation methodologies, which is an important consideration in radiomic studies. Additional file 3 describes the correlation of MTV with the extracted radiomic features.

\section{Discussion}

Radiomic research aims to improve the prediction of patient outcome through the extraction of additional data from medical images. However, numerous challenges with the extraction of radiomic features have been highlighted [10]. Selection of significant features for prognostic models is of considerable importance because external parameters such as delineation method and image reconstruction parameters affect reproducibility and robustness of these features $[14,36]$.

In this study, first, second and higher-order radiomic features were extracted from each of the PET-AS delineations. The significant variables in the developed prognostic models were dependent upon the delineation method. In the GCM3-based prognostic models, firstorder features kurtosis and skewness were found to be significant predictors of survival. However, higher-order feature GLNU was found to be of significance in combination with the first-order feature skewness in the WT-based prognostic model. For the AT and KM2based prognostic models, radiomic features were not found to be significant predictors of survival in comparison to the currently known predictors such as clinical stage and age. This highlights the dependency of significant PET radiomic variables on segmentation method.

Our findings demonstrate the potential impact of different segmentation methods for prognostic models using standardised implementations of radiomic features within clinical practice. Patients may be assigned different risk stratification groups depending on the segmentation method used in the process of developing the prognostic model. This could lead to sub-groups of patients receiving a more aggressive treatment than is necessary, leading to decreased quality of life. Furthermore, patients could potentially be denied beneficial treatment.

Nine segmentation methods were included in this study, with five being excluded from analysis after being reviewed by a radiologist. These methods were excluded due to poor tumour representation in a number of cases. In Additional file 1, the prognostic models developed from PET-AS methods that were excluded from the study are described. Interestingly, the excluded PET-AS methods FCM2, KM3, KM4 and RG developed identical prognostic models to the included methods AT and KM2. This suggests that whilst radiomic features are dependent upon the delineation method, this may be unrelated to the delineation method considered acceptable by a radiologist.
It has been reported that the accuracy of the segmentation delineation of the MTV is dependent upon tumour characteristics [9, 21, 24]. GCM-based segmentation methods have been shown to have limited performance in low TBR scenarios [24]. Furthermore, clustering methods such as FCM are highly dependent upon the heterogeneity of the tumour volumes. In homogeneous regions with low TBRs, the iterative process of FCM has been shown to overestimate the tumour volume [37]. Statistical-based segmentation algorithms such as RG compare adjoining voxel intensities. If the voxels are of similar intensities, they are included within the volume [38]. However, the performance of statistical-based RG segmentation algorithms in highly heterogeneous tumour volumes is degraded. Moreover, the performance of RG is dependent upon the defined stopping criteria. In our study, the RG algorithm stopped voxel inclusion when after an iteration no more than $5 \%$ of the total number of voxels already defined as the MTV were included. This stopping criteria has been reported to be limited in complex tumours [24].

This study used radiomic data derived using SUV bins of 0.5 units. In Additional file 2, the variance of radiomic features derived using different discretisation methods is shown but lies outside the scope of this study, so further analysis was not performed. Future work could investigate how different discretisation methods influences the significance of radiomic features in the development of prognostic models and subsequent impact on risk stratification in patients with OC.

The variability in segmentation performance in any one single clinical case means the standardisation of the delineation of the MTV is critical for the application of radiomics within OC. This supports the recommendations of the International Atomic Energy Agency (IAEA) whom state that there are no validated quantitative approaches for PET contouring that will result in ideal tumour delineation for all patients and tumour locations [39]. In addition, the American Association of Physicist in Medicine (AAPM) Task Group No 211 reported that they could not recommend a single PET auto segmentation method for MTV delineation. However, machine-learned segmentation methods have showed promise for accurate MTV delineation [9]. Machine-learned-based and consensus-based segmentation methodologies have been proposed for the standardisation of the delineation of the MTV [20, 40, 41]. In Additional file 3, radiomic features derived from each segmentation method were correlated with MTV. As described, GLNU, Energy and Coarseness were correlated with MTV for all PET segmentation methods. However, the level of correlation varied between PET-AS methods. Our study suggests that a standardised segmentation methodology should be used for the development of prognostic models.

Shape metrics can also be quantified from the primary tumour. Within this group of radiomic features, the 
surface to volume ratio (S2VR), sphere to volume ratio, compactness, sphericity and disproportion of the tumour can be characterised but have not been included in this study which focused on intra-tumoural heterogeneity. However, studies have investigated the inclusion of shape metrics in prognostic models [42].

The results of this study are strengthened by the large cohort $(n=427)$ of OC patients with contours assessed and approved by a Clinical Radiologist. The approach of controlling model development by using identical clinical data and standardised image features ensured that differences in risk stratification were due to the image segmentation method. In this study, we did not use any PET image interpolation algorithm before image feature analysis [34]. This approach however, is consistent with currently reported studies.

\section{Conclusion}

Prognostic models incorporating quantitative image features are dependent on the method used to delineate the primary tumour. This has a subsequent effect on risk stratification, with patients changing groups depending on the image segmentation method used. The standardisation of PET segmentation is important and should be considered in future prognostic and predictive clinical models. The findings of this study may have substantial potential impact on clinical management of patients with OC.

\section{Additional files}

Additional file 1: Prognostic models developed from PET autosegmentation methods excluded from the study. (DOCX $122 \mathrm{~kb}$ )

Additional file 2: Differences in radiomic features between two discretisation methods. (DOCX 69 kb)

Additional file 3: Correlation of radiomic features and the delineated Metabolic Tumour Volume. (DOCX 2375 kb)

\section{Acknowledgements}

Not applicable.

Avaliability of data and materials

Please contact author for data requests.

\section{Funding}

This work was partially funded by EPSRC-DTP grant ref. EP/M507842/1, Velindre NHS Trust grant number 2016/1 and Tenovus Cancer Care (TIG2016/04).

\section{Authors' contributions}

CP concieved the study, participated in data collection and drafted the manuscript. KF participated in data collection and analysis of results and helped draft the manuscript. PW participated in data collection. $\mathrm{RH}$ helped with statistical metholodogy and analysis of results. AR participated in the design of the study and helped draft the manuscript. CM participated in the design of the study and helped draft the manuscript. JS participated in the design of the study and helped draft the manuscript. ES participated in the design of the study, helped draft the manuscript and coordinated the study. All authors read and approved the final manuscript.

\section{Ethics approval and consent to participate}

All procedures performed in studies involving human participants were in accordance with the ethical standards of the instituitional and/or national research committee and with the 1964 Helsinki declaration and its later amendments or comparable ethical standards. Institutional Review Board approval was granted and requirement for informed consent was waived.

\section{Consent for publication}

Not applicable.

\section{Competing interests}

The authors declare they have no competing interests.

\section{Publisher's Note}

Springer Nature remains neutral with regard to jurisdictional claims in published maps and institutional affiliations.

\section{Author details}

'School of Engineering, Cardiff University, Queen's Buildings, 14-17 The Parade, Cardiff CF24 3AA, UK. ²Division of Cancer and Genetics, School of Medicine, UHW Main Building, Heath Park, Cardiff CF14 4XN, UK. ${ }^{3}$ Clinical Trials Unit, Cardiff University, Cardiff CF10 3AT, UK. ${ }^{4}$ Clinical Radiology, University Hospital of Wales, Heath Park, Cardiff CF14 4XW, UK. ${ }^{5}$ Wales Research and Diagnostic PET Imaging Centre, Cardiff University, School of Medicine, Ground Floor, C Block, UHW Main Building, Heath Park, Cardiff CF14 4XN, UK. ${ }^{6}$ Velindre Cancer Centre, Velindre Rd, Cardiff CF14 2TL, UK.

Received: 2 December 2017 Accepted: 23 March 2018

Published online: 11 April 2018

\section{References}

1. Oesophageal cancer survival statistics | Cancer Research UK. Oesophageal Cancer Surviv Stat Read More http//:www.cancerresearchuk.org/healthProfessional/cancer-Statistics/statistics-by-Cancer-Type/oesophageal-cancer/ survival\#Ftcako2Wk3QIZMFX99 2014. http://www.cancerresearchuk.org/ health-professional/cancer-statistics/statistics-by-cancer-type/oesophagealcancer/survi. Accessed 15 Mar 2017.

2. Teoh AYB, Chiu PWY, Yeung WK, Liu SYW, Wong SKH, Ng EKW. Long-term survival outcomes after definitive chemoradiation versus surgery in patients with resectable squamous carcinoma of the esophagus: results from a randomized controlled trial. Ann Oncol. 2013;24:165-71. https://doi.org/10.1093/annonc/mds206.

3. Corvò R. Evidence-based radiation oncology in head and neck squamous cell carcinoma. Radiother Oncol. 2007:85:156-70. https://doi.org/10.1016/j. radonc.2007.04.002

4. Moons KGM, Altman DG, Vergouwe Y, Royston P. Prognosis and prognostic research: application and impact of prognostic models in clinical practice. BMJ. 2009;338:b606. https://doi.org/10.1136/bmj.b606.

5. Vallieres M, Zwanenburg A, Badic B, Cheze-Le Rest C, Visvikis D, Hatt M. Responsible Radiomics research for faster clinical translation. J Nucl Med. 2017:jnumed.117.200501. https://doi.org/10.2967/jnumed.117.200501.

6. Hatt M, Majdoub M, Vallières M, Tixier F, Cheze-Le Rest C, Groheux D, et al. 18F-FDG PET uptake characterization through texture analysis: investigating the complementary nature of heterogeneity and functional tumor volume in a multi-cancer site patient cohort. J Nucl Med. 2015;56:38-44. https://doi. org/10.2967/jnumed.114.144055.

7. Yue Y, Osipov A, Fraass B, Sandler H, Zhang X, Nissen N, et al. Identifying prognostic intratumor heterogeneity using pre- and post-radiotherapy $18 \mathrm{~F}$ FDG PET images for pancreatic cancer patients. J Gastrointest Oncol. 2017;8: 127-38. https://doi.org/10.21037/jgo.2016.12.04.

8. Zaidi H, El Naqa I. PET-guided delineation of radiation therapy treatment volumes: a survey of image segmentation techniques. Eur J Nucl Med Mol Imaging. 2010;37:2165-87. https://doi.org/10.1007/s00259-010-1423-3.

9. Hatt M, Lee JA, Schmidtlein CR, El Naqa I, Caldwell C, De Bernardi E, et al. Classification and evaluation strategies of auto-segmentation approaches for PET: report of AAPM task group no. 211. Med Phys. 2017;44:e1-42. https:// doi.org/10.1002/mp.12124

10. Hatt M, Tixier F, Pierce L, Kinahan PE, Le Rest CC, Visvikis D. Characterization of PET/ CT images using texture analysis: the past, the present... any future? Eur J Nucl Med Mol Imaging. 2017;44:151-65. https://doi.org/10.1007/s00259-016-3427-0.

11. Yip SSF, Aerts HJWL. Applications and limitations of radiomics. Phys Med Biol. 2016;61:R150-66. https://doi.org/10.1088/0031-9155/61/13/R150. 
12. Hatt M, Tixier F, Cheze Le Rest C, Pradier O, Visvikis D. Robustness of intratumour 18F-FDG PET uptake heterogeneity quantification for therapy response prediction in oesophageal carcinoma. Eur J Nucl Med Mol Imaging. 2013:40:1662-71. https://doi.org/10.1007/s00259-013-2486-8.

13. Ben Bouallègue F, Al TY, Kafrouni M, Cartron G, Vauchot F, Mariano-Goulart D. Association between textural and morphological tumor indices on baseline PET-CT and early metabolic response on interim PET-CT in bulky malignant lymphomas. Med Phys. 2017:44:4608-19. https:/doi.org/10.1002/mp.12349.

14. Hatt M, Laurent B, Fayad H, Jaouen V, Visvikis D, Le Rest CC. Tumour functional sphericity from PET images: prognostic value in NSCLC and impact of delineation method. Eur J Nucl Med Mol Imaging. 2017; https:// doi.org/10.1007/s00259-017-3865-3.

15. Foley KG, Hills RK, Berthon B, Marshall C, Parkinson C, Lewis WG, et al. Development and validation of a prognostic model incorporating texture analysis derived from standardised segmentation of PET in patients with oesophageal cancer. Eur Radiol. 2018;28:428-36. https://doi.org/10.1007/s00330-017-4973-y.

16. Sobin L, Gospodarowicz MK, Wittekind C. UICC. TNM classification of malignant tumours. New York: Wiley; 2009.

17. Vinod SK, Min M, Jameson MG, Holloway LC. A review of interventions to reduce inter-observer variability in volume delineation in radiation oncology. J Med Imaging Radiat Oncol. 2016;60:393-406. https://doi.org/10. 1111/1754-9485.12462

18. Jiang J, Wu H, Huang M, Wu Y, Wang Q, Zhao J, et al. Variability of gross tumor volume in nasopharyngeal carcinoma using 11C-choline and 18F-FDG PET/CT. PLoS One. 2015;10:e0131801. https://doi.org/10.1371/journal.pone.0131801.

19. Greco C, Rosenzweig K, Cascini GL, Tamburrini O. Current status of PET/CT for tumour volume definition in radiotherapy treatment planning for nonsmall cell lung cancer (NSCLC). Lung Cancer. 2007;57:125-34. https://doi. org/10.1016/j.lungcan.2007.03.020

20. Schaefer A, Vermandel M, Baillet C, Dewalle-Vignion AS, Modzelewski R, Vera $P$, et al. Impact of consensus contours from multiple PET segmentation methods on the accuracy of functional volume delineation. Eur J Nucl Med Mol Imaging. 2016;43:911-24. https://doi.org/10.1007/s00259-015-3239-7.

21. Xu W, Yu S, Ma Y, Liu C, Xin J. Effect of different segmentation algorithms on metabolic tumor volume measured on 18F-FDG PET/CT of cervical primary squamous cell carcinoma. Nucl Med Commun. 2017;38:259-65. https://doi.org/10.1097/MNM.0000000000000641.

22. Orlhac F, Soussan M, Maisonobe J-AJ-A, Garcia CA, Vanderlinden B, Buvat I. Tumor texture analysis in 18F-FDG PET: relationships between texture parameters, histogram indices, standardized uptake values, metabolic volumes, and Total lesion Glycolysis. J Nucl Med. 2014;55:414-22. https://doi.org/10.2967/jnumed.113.129858.

23. van Velden FHP, Kramer GM, Frings V, Nissen IA, Mulder ER, de Langen AJ, et al. Repeatability of radiomic features in non-small-cell lung Cancer [18F]FDG-PET/CT studies: impact of reconstruction and delineation. Mol Imaging Biol. 2016;18:788-95. https://doi.org/10.1007/s11307-016-0940-2.

24. Berthon B, Marshall C, Evans M, Spezi E. Evaluation of advanced automatic PET segmentation methods using nonspherical thin-wall inserts. Med Phys. 2014;41:22502. https://doi.org/10.1118/1.4863480

25. Leijenaar RTH, Nalbantov G, Carvalho S, van Elmpt WJC, Troost EGC, Boellaard $R$, et al. The effect of SUV discretization in quantitative FDG-PET Radiomics: the need for standardized methodology in tumor texture analysis. Sci Rep. 2015;5:11075. https://doi.org/10.1038/srep11075.

26. Davies AR, Gossage JA, Zylstra J, Mattsson F, Lagergren J, Maisey N, et al. Tumor stage after neoadjuvant chemotherapy determines survival after surgery for adenocarcinoma of the esophagus and esophagogastric junction. J Clin Oncol. 2014;32:2983-90. https://doi.org/10.1200/JCO.2014.55.9070.

27. Tixier F, Le Rest CC, Hatt M, Albarghach N, Pradier O, Metges J-P, et al. Intratumor heterogeneity characterized by textural features on baseline 18FFDG PET images predicts response to concomitant radiochemotherapy in esophageal cancer. J Nucl Med. 2011;52:369-78. https://doi.org/10.2967/ jnumed.110.082404.

28. Yip C, Landau D, Kozarski R, Ganeshan B, Thomas R, Michaelidou A, et al Primary esophageal cancer: heterogeneity as potential prognostic biomarker in patients treated with definitive chemotherapy and radiation therapy. Radiology. 2014;270:141-8. https://doi.org/10.1148/radiol.13122869.

29. Doumou G, Siddique M, Tsoumpas C, Goh V, Cook GJ. The precision of textural analysis in 18F-FDG-PET scans of oesophageal cancer. Eur Radiol. 2015:25:2805-12. https://doi.org/10.1007/s00330-015-3681-8.

30. Gillies RJ, Kinahan PE, Hricak H. Radiomics: images are more than pictures, they are data. Radiology. 2016;278:563-77. https://doi.org/10.1148/radiol. 2015151169.
31. Yip C, Davnall F, Kozarski R, Landau DB, Cook GJR, Ross P, et al. Assessment of changes in tumor heterogeneity following neoadjuvant chemotherapy in primary esophageal cancer. Dis Esophagus. 2015;28:172-9. https://doi.org/ 10.1111/dote.12170

32. Nakajo M, Jinguji M, Nakabeppu Y, Nakajo M, Higashi R, Fukukura Y, et al. Texture analysis of 18F-FDG PET/CT to predict tumour response and prognosis of patients with esophageal cancer treated by chemoradiotherapy. Eur J Nucl Med Mol Imaging. 2017:44:206-14. https:// doi.org/10.1007/s00259-016-3506-2.

33. Amadasun M, King R. Textural features corresponding to textural properties. IEEE Trans Syst Man Cybern. 1989;19:1264-74. https://doi.org/10.1109/21.44046.

34. Depeursinge A, Foncubierta-Rodriguez A, Van De Ville D, Müller H. Threedimensional solid texture analysis in biomedical imaging: review and opportunities. Med Image Anal. 2014;18:176-96. https://doi.org/10.1016/j. media.2013.10.005.

35. Moons KGM, Kengne AP, Woodward M, Royston P, Vergouwe Y, Altman DG, et al. Risk prediction models: I. Development, internal validation, and assessing the incremental value of a new (bio)marker. Heart. 2012;98:68390. https://doi.org/10.1136/heartjnl-2011-301246.

36. Apostolova I, Rogasch J, Buchert R, Wertzel H, Achenbach HJ, Schreiber J, et al. Quantitative assessment of the asphericity of pretherapeutic FDG uptake as an independent predictor of outcome in NSCLC. BMC Cancer. 2014;14:110. https://doi.org/10.1186/1471-2407-14-896.

37. Hatt M, Cheze le Rest C, Turzo A, Roux C, Visvikis D. A fuzzy locally adaptive Bayesian segmentation approach for volume determination in PET. IEEE Trans Med Imaging. 2009:28:881-93. https://doi.org/10.1109/TMI.2008.2012036.

38. Monteiro ALR, Machado AMC, Lewer MHM. A Multicriteria method for cervical tumor segmentation in positron emission tomography. 2014 IEEE 27th Int. Symp. Comput. Med. Syst., IEEE; 2014, p. 205-8. doi:10.1109/CBMS.2014.52.

39. Konert T, Vogel W, MacManus MP, Nestle U, Belderbos J, Grégoire V, et al. PET/ CT imaging for target volume delineation in curative intent radiotherapy of non-small cell lung cancer: IAEA consensus report 2014. Radiother Oncol. 2015; 116:27-34. https://doi.org/10.1016/j.radonc.2015.03.014.

40. Berthon B, Marshall C, Evans M, Spezi E. ATLAAS: an automatic decision tree-based learning algorithm for advanced image segmentation in positron emission tomography. Phys Med Biol. 2016;61:4855-69. https://doi. org/10.1088/0031-9155/61/13/4855

41. Berthon B, Evans M, Marshall C, Palaniappan N, Cole N, Jayaprakasam V, et al. Head and neck target delineation using a novel PET automatic segmentation algorithm. Radiother Oncol. 2017;122:242-7. https://doi.org/ 10.1016/j.radonc.2016.12.008.

42. Desseroit MC, Visvikis D, Tixier F, Majdoub M, Perdrisot R, Guillevin R, et al. Development of a nomogram combining clinical staging with18F-FDG PET/ CT image features in non-small-cell lung cancer stage I-III. Eur J Nucl Med Mol Imaging. 2016;43:1477-85. https://doi.org/10.1007/s00259-016-3325-5.

43. Jentzen W, Freudenberg L, Eising EG, Heinze M, Brandau W, Bockisch A. Segmentation of PET volumes by iterative image thresholding. J Nucl Med. 2007:48:108-14

44. Drever L, Roa W, McEwan A, Robinson D. Iterative threshold segmentation for PET target volume delineation. Med Phys. 2007;34:1253-65. https://doi. org/10.1118/1.2712043

45. Day E, Betler J, Parda D, Reitz B, Kirichenko A, Mohammadi S, et al. A region growing method for tumor volume segmentation on PET images for rectal and anal cancer patients. Med Phys. 2009:36:4349-58. https://doi.org/10. $1118 / 1.3213099$

46. Belhassen S, Zaidi H. A novel fuzzy C-means algorithm for unsupervised heterogeneous tumor quantification in PET. Med Phys. 2010;37:1309-24. https://doi.org/10.1118/1.3301610.

47. Geets X, Lee JA, Bol A, Lonneux M, Grégoire V. A gradient-based method for segmenting FDG-PET images: methodology and validation. Eur J Nucl Med Mol Imaging. 2007:34:1427-38. https://doi.org/10.1007/s00259-006-0363-4.

48. Tylski P, Bonniaud G, Decenciere E, Stawiaski J, Coulot J, Lefkopoulos D, et al. 18F-FDG PET images segmentation using morphological watershed: a phantom study. 2006 IEEE Nucl. Sci. Symp. Conf. Rec., vol. 4, IEEE; 2006, p. 2063-2067. doi:https://doi.org/10.1109/NSSMIC.2006.354319. 